\title{
The Effect of Elaeagnus Angustifolia Extract on the Joint Friction and Antioxidant Activity in Knee Non-Traumatic Osteoarthritis Model in Rat
}

\author{
M. Mofid ${ }^{1,2}$, S. Homayoon Sadraie ${ }^{1}$, H. Imani ${ }^{1}$, G. Torkaman ${ }^{3}$, G. Kaka ${ }^{1}$, M. Reza \\ Naghii ${ }^{4}$, G. Alishiri ${ }^{5}$ \\ ${ }^{1}$ Department of Anatomical Sciences, Faculty of Medicine, Baqiyatallah University of Medical Sciences, Tehran, Iran \\ ${ }^{2}$ Neuroscience research center, Baqiyatallah University of Medical Sciences, Tehran, Iran \\ ${ }^{3}$ Department of Physiotherapy, Faculty of Medical Sciences, Tarbiat Modares University, Tehran, Iran \\ ${ }^{4}$ Department of Nutrition, Health School, Baqiyatallah University of Medical Sciences, Tehran, Iran. \\ ${ }^{5}$ Department of Rheumatology, Faculty of Medicine, Baqiyatallah University of Medical Sciences, Tehran, Iran
}

\section{CORRESPONDING AUTHOR:}

Giti Torkaman

Tarbiat Modares University

Ale-Ahmad Street

Tehran, Iran

E-mail: torkamg@modares.ac.ir

DOI:

10.32098/mltj.03.2020.05

LEVEL OF EVIDENCE: Animal Study

\begin{abstract}
SUMMARY
Object. The objective was to investigate the effect of Elaeagnus angustifolia (E. angustifolia) extract on the joint lubrication and the activity of antioxidant in non-traumatic knee osteoarthritis model in rat.

Methods. In this study, 28 male albino Wistar rats were randomly assigned into four groups, each comprised of seven rats: Control (healthy animals); Saline (intra-articular injection of 50 $\mu \mathrm{L}$ Saline); monoidoacetate, MIA (intra-articular injection of $50 \mu \mathrm{L} \mathrm{MIA(;} \mathrm{and} \mathrm{MIA+extract}$ (intra-articular injection of MIA and $500 \mathrm{mg} / \mathrm{kg}$ daily of E. angustifolia extract intraperitoneally for four weeks). Osteoarthritis was induced by injection of $50 \mu \mathrm{L}$ solution of $3 \mathrm{mg}$ MIA in rats through infrapatellar ligament. Six weeks after intra-articular injection, Joint friction parameters consisting of cycle number, maximum peak, exponential and linear slope of cycle decay, and coefficient of friction) COF) were measured in knee joint. Activity of the superoxide dismutase (SOD), malondialdehyde (MDA) and glutathione peroxidase (GPx) were determined in blood plasma.

Results. MIA injection reduced the cycle number of joint oscillation compared to the Control group. In the MIA+extract group, cycle number and maximum peak increased significantly compared to the MIA group ( $\mathrm{P}=0.032$ and $\mathrm{P}=0.016$, respectively). E. angustifolia extract resulted in the increase of SOD and GPx activity $(\mathrm{P}=0.011$ and $\mathrm{P}=0.05$, respectively) and the decrease of MDA activity ( $\mathrm{P}=0.000)$ compared to MIA group.

Conclusions. The results showed that E. angustifolia extract is effective to improve the antioxidant enzyme activity and subsequently to decrease the joint friction in the non-traumatic knee osteoarthritis model.
\end{abstract}

\section{KEY WORDS}

Elaeagnus angustifolia extract; knee osteoarthritis; joint friction; Superoxide dismutase (SOD); Malondialdebyde (MDA); Glutathione peroxidase (GPx); rat.

\section{INTRODUCTION}

Osteoarthritis (OA) is the most common joint disease in adults, and its prevalence increases with age, causing disability in the elderly population (1). OA is a multifactorial disorder of synovial joints, which is characterized by escalated degeneration and loss of articular cartilage(2). Hyaline cartilage in the diarthroidal joints is serving as a low friction, wear resistant surface for load support, load transfer and joint motion (3).

Proposed mechanisms including hydrodynamic lubrication, boundary lubrication, elastic deformation, and fluid pressurization are responsible for maintaining the low-friction and good lubrication in the diarthrodial joint (4). The excessive friction accelerates cartilage wear after failure of carti- 
lage lubrication. Damage of cartilage may be a key factor in the onset of osteoarthritis (5).

The joint coefficient of friction (COF) is thought to increase with osteoarthritis progression, and this increase may occur due to a decrease of lubricants concentration such as lubricin (6).

Lubricin as a glycoprotein, thought to be a boundary lubricant in synovial fluid and articular cartilage (7) is deficient in aspirates of acute post-traumatic knee effusions, which is likely to be result of inflammatory destruction (7).

Friction measurements are important to determine the relative tribological contributions made by synovial fluid or cartilage (8) and/or to assess the efficacy of therapeutic modalities for preventing the development of osteoarthritis (9).

Evidence from both experimental and clinical studies suggests that oxidative stress plays an important role in the pathogenesis of $\mathrm{OA}(10,11)$. It is indicated that the oxidant parameters including total peroxide (TP), lipid hydroperoxide and Oxidative stress index (OSI) increased and antioxidant parameters such as plasma total antioxidant capacity (TAC), thiol level, catalase activity and prolidase activity decreased in patients with osteoarthritis; therefore, these patients may be exposed to a potent oxidative stress (12). Malondialdehyde (MDA) and superoxide dismutase (SOD) have often been treated as matching indicators, of which the SOD level is associated with free radical scavenging ability, whereas MDA reflects the degree of damage caused by free radicals to the cells. When the level of free radicals was high, it was found that SOD failed to effectively remove them, leading to lipid peroxidation and cell damage (13).

The extracts of leaves and flowers of E. angustifolia leaves contain phenolic and flavonoid compounds which have antioxidant properties and protect cells from oxidative damages and delays or reduces the risk of many degenerative diseases. It has shown that the amounts of phenolic and flavonoid compounds are higher in leaves than flower of the plant. The explanation might be due to the process of photosynthesis which occurs in the leaves and also the existence of high amount of flavonoid biosynthetic pathway precursors in the leaves compared to any other organs (14). $E$. angustifolia leaves contain various chemical compounds including amino acids, flavonoids, phenolic compounds, polysaccharides and some other essential elements (15). The polysaccharides are important component of E. angustifolia leaves with anti-radiation, antioxidant and immune regulatory activities (16).The anti-inflammatory effects of the fruits of this plant on rheumatoid arthritis and osteoarthritis have been reported (17).
Hence, the aim of this study was to investigate the effect of E. angustifolia extract on the joint friction and the activity of antioxidants in the non-traumatic OA model in rats.

\section{MATERIALS AND METHODS}

This research was carried out according to the internationally valid guidelines and the institutional animal ethics committee in Baqiyatallah University of Medical Sciences. In this study, 28 adult male albino Wistar rats (12-14 weeks age and weighing 200-250 g) were purchased from the laboratory animal center of the university. The rats were kept under standard housing laboratory conditions (room temperature of $23 \pm 2{ }^{\circ} \mathrm{C}$ with relative humidity of $60 \pm 5 \%$, and $12 \mathrm{hr} / 12 \mathrm{hr}$ light/dark cycles) and were fed with a standard laboratory pellets rat chow and water ad libitum. One week after arrival and adaptation, rats were randomly divided into four groups. Each group comprised of seven rats and were observed regularly. Control group (consisted of healthy animals that underwent no intervention); Saline group $(50 \mu \mathrm{L}$ of normal Saline $0.9 \%$, injected into the right knee joint as a placebo group); Monoiodoacetate group (MIA group, received $50 \mu \mathrm{L}$ solution containing $3 \mathrm{mg}$ MIA into their right knee joint); and MIA+ E. angustifolia extract group (MIA+ extract group, MIA injection and $500 \mathrm{mg} / \mathrm{kg}$ body weight daily E. angustifolia extract injection intraperitoneally for four weeks (12).

\section{Method of osteoarthritis induction}

Osteoarthritis was induced by intra-articular injection of MIA. MIA injection is considered to induce osteoarthritis in joint by damaging glycolysis in the joint, resulting in the eventual death of chondrocytes by inhibiting glyceraldehyde-3-phosphate dehydrogenase activity and increasing inflammation (18). First, the rats of MIA and MIA+extract groups were anesthetized by using Ketamine $(50 \mathrm{mg} / \mathrm{kg})$ and Xylazine $(5 \mathrm{mg} / \mathrm{kg})$. After shaving the knee skin and bending the knee joint, a single dose of $3 \mathrm{mg}$ MIA (Sigma-Aldrich, St. Louis, MO) dissolved in $50 \mu \mathrm{L}$ physiological Saline $(0.9 \%)$ was injected through infrapatellar ligament of the right knee using a 26-gauge needle. In the Saline group, by using a 26-gauge needle, $50 \mu \mathrm{L}$ Saline $0.9 \%$ solution was injected into the right knee joint capsule through infrapatellar ligament. At 43 th day, all animals in four groups were euthanized by overdose of the anesthetic drugs and were sacrificed to measure the joint friction. For blood plasma analyses, blood samples obtained from anaesthetized mice were centrifuged immediately (Megafuge 1.0 Heraeus Sepatech $\mathrm{GmbH}$ ) at $3000 \mathrm{rpm}$ for $15 \mathrm{~min}$ at $+4{ }^{\circ} \mathrm{C}$ to determine the activity of 
superoxide dismutase (SOD), malondialdehyde (MDA) and glutathione peroxidase (GPx).

\section{Method of preparation and injection of $E$. angustifolia extract}

The fruits of E. angustifolia were collected from mountain area of Niavaran, Tehran, Iran and was identified and approved by Herbarium Department of Pharmacognosy of Shahid Beheshti University of Medical Sciences, Tehran, Iran. The voucher specimen [No. 1257] is preserved in the herbarium of this department for reference.

The aqueous extract was prepared by adding $1000 \mathrm{ml}$ of distilled water to $100 \mathrm{~g}$ of fruit powder (seed, flesh, and peel) and the resulting solution was boiled for 8-10 minutes. Then, the mixture was filtered and the solution was completely dehydrated for 8-10 hours in water bath to provide a crude extract with $20 \%$ yield. This extract was then weighed and after being dissolved in physiological Saline, and consequently 14 days after induced OA, $500 \mathrm{mg} / \mathrm{kg}$ was injected intraperitoneally in animals in the MIA+extract group, daily for four weeks.

\section{Joint friction (biotribological) measurement}

The knee joint was resected following transection of the middle of tibia/fibula and femoral shafts. Friction testing was performed immediately after the joint resection by a pendulum friction tester. The knee joint was placed in the free pendulum tester. Pendulum rotation was calculated for flexion-extension in the sagittal plane and other motion out of this plane was ignored (figure 1). Through an automated gated mechanism, movement was started from a fix point and free oscillation around the knee joint recorded. Ravanbod et al., 2011, report more technical details on the friction tester(19).

The pendulum load was approximately half of the body weight of animal. Angular changes were recorded to the equilibrium point at a sampling frequency of $30 \mathrm{~Hz}$. Cycle number to reach equilibrium, maximum peak oscillation value, exponential and linear slope of cycles decay were calculated with Labview 7.1 Software (National instrument Labview 7.1 software, Austin, TX, USA). Coefficient of friction (COF) was calculated according to Stanton's equation (19).
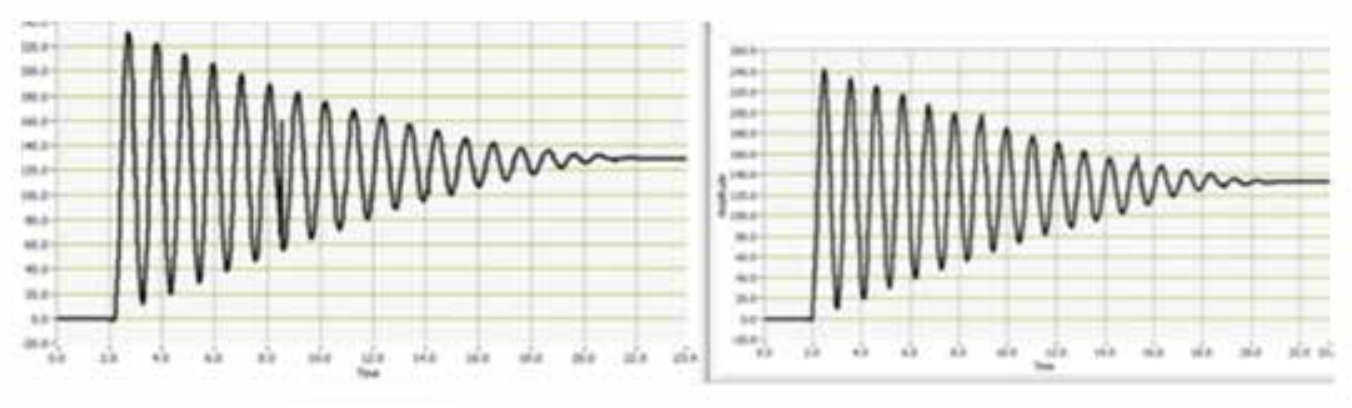

\section{Contorol}

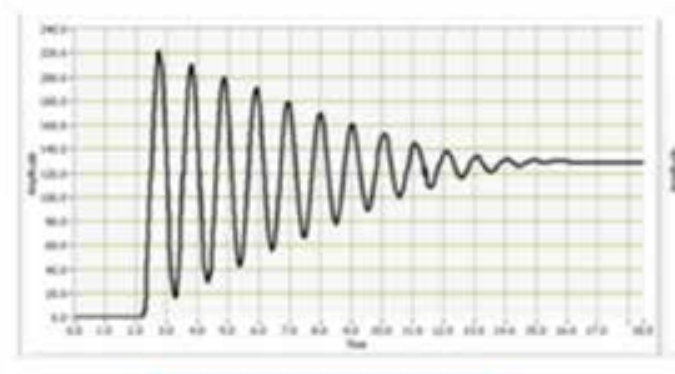

Saline

\section{MIA+extract}

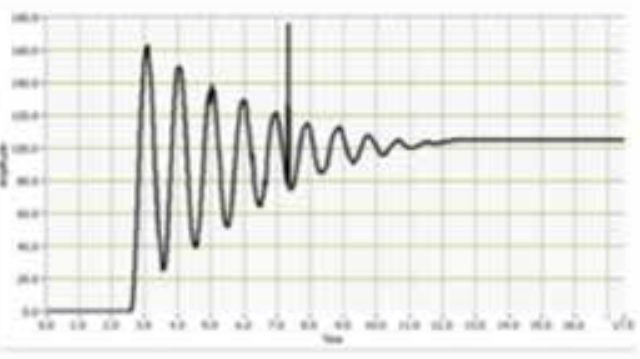

\section{MIA}

Figure 1. A sample of free oscillation in Control, Saline, MIA, and MIA+extract groups. 


\section{Determination of biochemical parameters}

SOD activity was determined according to Light spectroscopy method by Winterbourn et al. (20).

Glutathione peroxidase activity was determined by glutathione reductase enzyme coupling reaction according to method by Lushchak et al.(21). Reduction of glutathione oxide occurs by Gpx reaction with NADPH in the presence of glutathione reductase (GR). In this reaction, NADPH oxidation to $\mathrm{NADP}^{+}$reduces absorption at $340 \mathrm{~nm}$, which is proportional to GPx activity $\left(\mathrm{H}_{2} \mathrm{O}+\mathrm{GS}-\mathrm{SG} \rightarrow 2 \mathrm{GSH}+\right.$ $\mathrm{H}_{2} \mathrm{O}_{2}$ ). Malondialdehyde is the ultimate oxidizing peroxidase product in the body's lipid membrane cells. Thiobarbituric acid assay (TBA) (22) was used to measure MDA level.

\section{STATISTICAL ANALYSIS}

The Shapiro-Wilk test was used to determine the normal distribution of data. Whereas data did not follow a normal distribution $\mathrm{P}<0.05$, the non-parametric Kruskal-Wallis test and Mann-Whitney $\mathrm{u}$ test was used to analyze and compare the group differences. The statistical significance was defined as $\mathrm{P} \leq 0.05$. These statistical analyses were carried out using the SPSS for Windows Statistical Software Package.

\section{RESULTS}

\section{The joint friction parameters}

The measurements of joint friction parameters are shown in table I.

\section{Cycle number}

The cycle number to reach equilibrium showed a significant difference through the study groups $(\mathrm{P}=0.005)$. Cycle number in Saline, MIA, and MIA+extract groups decreased compared to Control group $(\mathrm{P}<0.05)$. After using the $E$. angustifolia extract, the cycle number in the MIA+extract group was significantly higher than MIA group ( $\mathrm{P}=0.032)$. An increase in the cycle number reflects the reduction of joint friction.

\section{Maximum peak}

The maximum peak oscillation showed a significant difference among the groups $(\mathrm{P}=0.002)$. The maximum Peak oscillation decreased significantly in Saline, MIA, and MIA+extract compared to Control group $(\mathrm{P}<0.05)$. After using the E. angustifolia extract, the maximum peak oscillation increased significantly compared to Saline group $(\mathrm{P}=0.016)$ and MIA group $(\mathrm{P}=0.016)$. Increasing the maximum peak reflects the facilitated movement in the joint and the reduction of joint friction.

\section{Exponential slope of cycle decay}

The exponential slope of cycle decay showed a significant difference among the groups $(\mathrm{P}=0.010)$. The exponential slope of oscillation showed an increase in all groups compared to Control group, but this increase was only significant in Saline group $(\mathrm{P}=0.008)$, and MIA group $(\mathrm{P}=0.008)$. After administration of the E. angustifolia extract, the exponential slope of the oscillation decreased and its value showed no significant difference compared to Control group. The decrease in the exponential slope of cycle decay indicates that the joint movement is performed with low friction.

\section{Linear slope of cycle decay}

The slope of cycle decay showed no significant difference among the groups $(\mathrm{P}=0.062)$.

\section{Joint friction coefficient}

The friction coefficient calculated by the Stanton formula showed no significant difference among the groups $(\mathrm{P}=$ $0.131)$.

Table I. The value of joint friction parameters (Mean \pm SD)

\begin{tabular}{lllll}
\hline Variables & Control & Saline & MIA & MIA+extract \\
\hline Cycle Number & $20.60 \pm 1.51$ & $12.60 \pm 2.88^{\mathrm{a}}$ & $12.20 \pm 1.48^{\mathrm{a}}$ & $15.40 \pm 2.40^{\mathrm{ab}}$ \\
\hline Maximum Peak & $28.14 \pm 1.76$ & $21.38 \pm 1.03^{\mathrm{a}}$ & $21.35 \pm 1.34^{\mathrm{a}}$ & $24.07 \pm 0.85^{\mathrm{abc}}$ \\
\hline Exponential Slope & $0.04 \pm 0.007$ & $0.12 \pm 0.08^{\mathrm{a}}$ & $0.11 \pm 0.03^{\mathrm{a}}$ & $0.08 \pm 0.3$ \\
\hline Linear slope & $0.008 \pm 0.000$ & $0.011 \pm 0.002$ & $0.012 \pm 0.001$ & $0.010 \pm 0.001$ \\
\hline Friction coefficient & $0.24 \pm 0.01$ & $0.31 \pm 0.05$ & $0.26 \pm 0.15$ & $0.28 \pm 0.04$ \\
\hline
\end{tabular}

a: Significant decrease compared to Control group; b: Significant increase in the MIA+extract group compared to MIA group; c: Significant increase in the MIA+extract group compared to Saline group. 


\section{Biochemical parameters}

The measured biochemical variables including the activity of antioxidant enzymes: SOD, GPx and MDA as a lipid peroxidation index are shown in table II.

\section{Superoxide dismutase (SOD) activity}

The amount of SOD activity was significantly different in the groups $(\mathrm{P}=0.001)$. The induction of osteoarthritis using MIA reduced the amount of SOD activity. This decrease was significant in both MIA $(\mathrm{P}=0.000)$ and MIA+extract $(\mathrm{P}=0.025)$ groups compared to Control group. Using $E$. angustifolia extract increased the amount of SOD activity compared to MIA group $(\mathrm{P}=0.011)$.

\section{Glutathione peroxidase (GPx) activity}

The level of GPx activity revealed significant differences in the groups ( $\mathrm{P}=0.041)$, The GPx activity in the MIA+extract group was significantly higher than Control $(P=0.018)$, Saline $(\mathrm{P}=0.021)$, and MIA $(\mathrm{P}=0.05)$ groups.

\section{Malondialdehyde (MDA) activity}

The level of MDA activity showed significant differences in the groups $(\mathrm{P}=0.002)$. In MIA group, the level of MDA activity increased compared to Control and Saline groups; this increase was significant in Saline group $(\mathrm{P}=0.031)$. The level of MDA activity in the MDA+extract group decreased compared to Control $(\mathrm{P}=0.002)$, Saline $(\mathrm{P}=0.005)$ and MIA $(\mathrm{P}=0.000)$ groups.

\section{DISCUSSION}

In this study, the effect of $E$ angustifolia extract was investigated on the SOD, GPx, and MDA activity and also the decrease of joint friction in the non-traumatic OA model. Although the measuring of friction is very important to determine of joint function, but it is an aggressive method that is not feasible in human studies. So, in this study we used MIA rat model to investigate the effect of $E$ angustifolia extract on the joint friction. The MIA model in rat, is well established and resembles the histological and pain-related behavior of human degenerative OA (23). Injection of MIA leads to a decreased number of chondrocytes and subsequent histological and morphological articular alterations similar to human osteoarthritic changes (23).

The results of this study revealed that induction of osteoarthritis by MIA injection reduced the cycle number of joint oscillation compared to Control group. In the MIA+extract group, after four weeks administration of E. angustifolia extract, the number cycle increased significantly compared to MIA group. The use of Saline also reduced the cycle number of oscillation, which was previously reported by Caligaris et al. (24). After using the E angustifolia extract, the maximum peak oscillation increased compared to the Saline and MIA groups. Administration of E. angustifolia extract displayed the decrease of exponential slop of cycle decay, and after treatment, no significant difference observed between MIA+extract group and Control group. Both linear slope of cycle decay and the friction coefficient showed no significant decrease in the groups. Crisco et al., (2007) showed that exponential slope of cycle decay is more accurate than the linear slop of cycle decay for predicting the joint friction coefficient (25). They suggest that the exponential model is capable to predict the friction coefficient accurately, when the viscous damping is quite small. In our study, exponential slop of cycle decay determined the difference of joint coefficient of friction among groups; whereas, both linear slop of cycle decay and the friction coefficient prediction by Stanton formula (based on the linear decay) could not determine any difference among groups.

Dunham et al., (1993) suggested that, "the intra-articular injection of MIA leads to inhibition of glyceraldehyde-3-phosphate dehydrogenase in chondrocytes, which causes interruption of glycolysis, increased cartilage destruction, as well as a decrease of proteoglycan synthesis and ultimately cell death" (18). It is noted that MIA injection causes cartilage destruction (9). Naveen et al., (2014) showed that, two weeks after induced OA by MIA, the ratio of glycosaminoglycan/total protein content and the cartilage stiffness were decreased (26).

The joint friction can be significantly changed in articular diseases even before appearance of clinical or laboratory

Table II. The measured biochemical parameters (Mean \pm SD)

\begin{tabular}{lllll}
\hline Variables & Control & Saline & MIA & MIA+extract \\
\hline SOD $(u m / 1)$ & $0.74 \pm 0.05$ & $0.73 \pm 0.03^{\mathrm{b}}$ & $0.61 \pm 0.04^{\mathrm{a}}$ & $0.65 \pm 0.07^{\mathrm{ab}}$ \\
\hline GPx $(\mathrm{u} / \mathrm{l})$ & $195.5 \pm 62.19$ & $190.3 \pm 36.31$ & $235.7 \pm 130.4$ & $401.7 \pm 186.9^{\mathrm{abc}}$ \\
\hline MDA(umol/l) & $4.49 \pm 1.19$ & $4.24 \pm 1.38$ & $5.71 \pm 0.72^{\mathrm{ab}}$ & $2.36 \pm 1.08^{\mathrm{abc}}$ \\
\hline
\end{tabular}

$\mathrm{a}$ :Significant difference with Control group, b: significant difference with MIA group, c: significant difference with Saline group 
symptoms (27). The interaction of articular cartilage and synovial fluid through the two important mechanisms of boundary lubrication and fluid film produces smooth and low friction movements in the normal synovial joints and causes performing of daily functional activities with minimal friction and good lubrication to prevent the joint cartilage wearing. Some studies have shown that there is a direct correlation between the increase of friction coefficient and the intensity of OA (28-30).

The experimental and clinical studies suggest that oxidative stress may be considered as one of the main etiological factors in the pathogenesis of OA $(10,11,31)$. SOD expression is significantly down regulated in OA chondrocytes in vivo, but this precedes the development of OA lesions, raising the possibility that alterations in SOD expression are associated with the earliest stages of OA pathogenesis (32). E. angustifolia is consisting of various chemical compounds including amino acids, flavonoids, phenolic compounds, polysaccharides and some other essential elements (15). Most studies on E. angustifolia is related to its antioxidant capacity of phenolic compounds and antocyanosides. Polyphenols can suppress the oxidative stress produced by reactive oxygen species (ROS) with their hydroxyl groups $(33,34)$.

Our results showed that the intra-articular injection of MIA caused the decrease of SOD activity level. The level of SOD and GPx activities increased in MIA+extract group. The amount of GPx activity in the MIA+ extract group was significantly higher than Control, Saline, and MIA groups. Moreover, the amount of MDA activity increased in the MIA group compared to the Control and Saline groups. The level of MDA activity decreased significantly after administration of E. angustifolia extract in MIA+extract group compared to other groups. It was found that the intra-peritoneal injection of E. angustifolia extract was effective in increasing the level of activity of antioxidant enzymes (SOD and GPx) and decreasing the level of MDA activity. It is reported that antioxidants have been effective in altering the oxidative enzymes levels in osteoarthritis (35). Increased MDA and decreased glutathione (GSH), and catalase activities (CAT) were evident in OA patients (11). MDA or lipid peroxidation increased in plasma and synovial fluid of osteoarthritis and rheumatoid arthritis patients (11). Anti-

\section{REFERENCES}

1. Felson DT, Lawrence RC, Hochberg MC, McAlindon T, Dieppe PA, Minor MA, et al. Osteoarthritis: new insights. Part 2: treatment approaches. Ann Intern Med 2000;133(9):726-37.

2. Poole AR GF, Abramson SB. Etiopathogenesis of osteoarthritis. In:Osteoarthritis. Diagnosis and Medical/Surgical Manage- oxidant enzymes, such as SOD, CAT, GPx are decreased in OA patients, confirming the role of oxidative stress in $\mathrm{OA}$ pathogenesis $(10,12)$.

In the non-traumatic OA model, Maghzi et al., (2015) showed that the oral treatment with E. angustifolia extract decreased the Mankin score (glycosaminoglycan index) and cellular degradation in articular cartilage (36).

Based on the effectiveness of E. angustifolia extract on the prevention of chondroblast cell death, it may be facilitated the expression of joint lubricants such as glycoproteins and hyaluronic acid and subsequently decrease of joint friction. We could not measure the level of these lubricants in this study and it suggests to consider in future studies. It seems that the prevention of cartilage degradation and the synthesis of lubricant agents after intraperitoneal injection of $E$. angustifolia extract, may be effective for decreasing the joint friction and better lubrication during functional activity, but special histological and biochemical studies should be considered in the future studies.

\section{CONCLUSIONS}

Induction of osteoarthritis by MIA injection reduced the cycle number of joint oscillation. Administration of $E$. angustifolia extract decreased the exponential slop of cycle decay, and after treatment this parameter showed no significant difference compared to Control group. E. angustifolia extract was effective in increasing the level of activity of antioxidant enzymes (SOD and GPx) and decreasing the level of MDA activity. It seems that E. angustifolia extract through its antioxidant effect be able to increase the joint lubrication in the osteoarthritis.

\section{ACKNOWLEDGEMENTS}

We would like to express my special thanks of gratitude to Dr Roya Ravanbod (assistant professor of Tarbiat Modares University) to help for performing joint friction test.

\section{CONFLICT OF INTERESTS}

The authors declare that they have no conflict of interests.

ment. 4 ed. RW Moskowitz RA, MC Hochberg, JA Buckwalter, VM.Goldberg, editor. Philadelphia Lippincott Williams \& Wilkins 2007.

3. Morlock M, Schneider E, Bluhm A, et al. Duration and frequency of every day activities in total hip patients. J Biomech 2001;34(7):873-81. 
4. Margareta Nordin DirSci VHF. Basic Biomechanics of the Musculoskeletal System. USA: LWW; Fourth, North American 2012.

5. Ballantine GC SG. The effect of lipid depletion on osteoarthritic wear. Wear. 2002(253):385-93.

6. Teeple E, Elsaid KA, Fleming BC, Jay GD, Aslani K, Crisco JJ, et al. Coefficients of friction, lubricin, and cartilage damage in the anterior cruciate ligament-deficient guinea pig knee. J Orthop Res 2008;26(2):231-7.

7. Jay GD, Elsaid KA, Zack J, et al. Lubricating ability of aspirated synovial fluid from emergency department patients with knee joint synovitis. J Rheumatol 2004;31(3):557-64.

8. Tanaka E, Iwabe T, Dalla-Bona DA, et al. The effect of experimental cartilage damage and impairment and restoration of synovial lubrication on friction in the temporomandibular joint. J Orofac Pain 2005;19(4):331-6.

9. Kawano T, Miura H, Mawatari T, et al. Mechanical effects of the intraarticular administration of high molecular weight hyaluronic acid plus phospholipid on synovial joint lubrication and prevention of articular cartilage degeneration in experimental osteoarthritis. Arthritis Rheum 2003;48(7):1923-9.

10. Ostalowska A, Birkner E, Wiecha M, et al. Lipid peroxidation and antioxidant enzymes in synovial fluid of patients with primary and secondary osteoarthritis of the knee joint. Osteoarthritis Cartilage 2006;14(2):139-45.

11. Surapaneni KM, Venkataramana G. Status of lipid peroxidation, glutathione, ascorbic acid, vitamin $\mathrm{E}$ and antioxidant enzymes in patients with osteoarthritis. Indian J Med Sci. 2007;61(1):9-14.

12. Altindag O, Erel O, Aksoy N, Selek S, Celik H, Karaoglanoglu $M$. Increased oxidative stress and its relation with collagen metabolism in knee osteoarthritis. Rheumatol Int 2007;27(4):339-44.

13. Yang X, Zhao J, He Y, Huangfu X. Screening for characteristic genes in osteoarthritis induced by destabilization of the medial meniscus utilizing bioinformatics approach. J Musculoskelet Neuronal Interact 2014;14(3):343-8.

14. Saboonchian F, Jamei R, Hosseini Sarghein S. Phenolic and flavonoid content of Elaeagnus angustifolia L. (leaf and flower). Avicenna J Phytomed 2014;4(4):231-8.

15. Amiri Tehranizadeh Z, Baratian A, Hosseinzadeh H.Russian olive (Elaeagnus angustifolia) as a herbal healer. Bioimpacts. 2016;6(3):155-67.

16. Chen Q, Chen J, Du H, Li Q, Chen J, Zhang G, et al. Structural characterization and antioxidant activities of polysaccharides extracted from the pulp of Elaeagnus angustifolia L Int J Mol Sci. 2014;15(7):11446-55.

17. Nikniaz Z, Ostadrahimi A, Mahdavi R, Ebrahimi AA, Nikniaz L Effects of Elaeagnus angustifolia L. supplementation on serum levels of inflammatory cytokines and matrix metalloproteinases in females with knee osteoarthritis. Complement Ther Med 2014;22(5):864-9.

18. Dunham J, Hoedt-Schmidt S, Kalbhen DA. Prolonged effect of iodoacetate on articular cartilage and its modification by an anti-rheumatic drug. Int J Exp Pathol 1993;74(3):283-9.

19. Ravanbod R, Torkaman G, Esteki A. Biotribological and biomechanical changes after experimental haemarthrosis in the rabbit knee. Haemophilia 2011;17(1):124-33.
20. Winterbourn CC, Hawkins RE, Brian M, Carrell RW. The estimation of red cell superoxide dismutase activity. J Lab Clin Med 1975;85(2):337-41.

21. Lushchak VI, Bagnyukova TV. Temperature increase results in oxidative stress in goldfish tissues. 2. Antioxidant and associated enzymes. Comp Biochem Physiol C Toxicol Pharmacol 2006;143(1):36-41.

22. Lapenna D, Ciofani G, Pierdomenico SD, Giamberardino MA, Cuccurullo F. Reaction conditions affecting the relationship between thiobarbituric acid reactivity and lipid peroxides in human plasma. Free Radic Biol Med 2001;31(3):3315.

23. Lampropoulou-Adamidou K, Lelovas P, Karadimas EV, et al. Useful animal models for the research of osteoarthritis. Eur J Orthop Surg Traumatol 2014;24(3):263-71.

24. Caligaris M, Canal CE, Ahmad CS, et al. Investigation of the frictional response of osteoarthritic human tibiofemoral joints and the potential beneficial tribological effect of healthy synovial fluid. Osteoarthritis Cartilage 2009;17(10):1327-32.

25. Crisco JJ, Blume J, Teeple E, et al. Assuming exponential decay by incorporating viscous damping improves the prediction of the coefficient of friction in pendulum tests of whole articular joints. Proc Inst Mech Eng H 2007;221(3):325-33.

26. Naveen SV, Ahmad RE, Hui WJ, et al. Histology, glycosaminoglycan level and cartilage stiffness in monoiodoacetate-induced osteoarthritis: comparative analysis with anterior cruciate ligament transection in rat model and human osteoarthritis. Int J Med Sci 2014;11(1):97-105.

27. Katta J, Jin Z, Ingham E, Fisher J. Biotribology of articular cartilage--a review of the recent advances. Med Eng Phys 2008;30(10):1349-63.

28. Elsaid KA, Jay GD, Warman ML, et al. Association of articular cartilage degradation and loss of boundary-lubricating ability of synovial fluid following injury and inflammatory arthritis. Arthritis Rheum.2005;52(6):1746-55.

29. Jay GD, Fleming BC, Watkins BA, et al. Prevention of cartilage degeneration and restoration of chondroprotection by lubricin tribosupplementation in the rat following anterior cruciate ligament transection. Arthritis Rheum 2010;62(8):2382-91.

30. Teeple E, Elsaid KA, Jay GD, et al. Effects of supplemental intra-articular lubricin and hyaluronic acid on the progression of posttraumatic arthritis in the anterior cruciate ligament-deficient rat knee. Am J Sports Med 2011;39(1):164-72.

31. Sutipornpalangkul W, Morales NP, Charoencholvanich K, et al. Lipid peroxidation, glutathione, vitamin $\mathrm{E}$, and antioxidant enzymes in synovial fluid from patients with osteoarthritis. Int J Rheum Dis 2009;12(4):324-8.

32. Scott JL, Gabrielides C, Davidson RK, et al. Superoxide dismutase downregulation in osteoarthritis progression and end-stage disease. Ann Rheum Dis 2010; 69(8):1502-10.

33. Wang Y GT, Zhao C, Zhao P. Changes in total phenolic and flavonoid contents and antioxidant activities of the fruit from Elaeagnus angustifolia during an 80-day study period Agro Food Ind Hi Tech 2014 (25):7-10.

34. Yalcin G SO. Antioxidant capacity of Elaeagnus angustifolia L. and investigation of eosin y as the fluorescent probe in ORAC method J Food Agric Environ 2014; (12):51-4 
35. Panahi Y, Rahimnia AR, Sharafi M, et al. Curcuminoid treatment for knee osteoarthritis: a randomized double-blind placebo-controlled trial. Phytother Res 2014;28(11):1625-31.
36. Maghzi M, Khorsandi L, Orazizadeh M, et al. Histological effects of Elaeagnus angustifolia aqueous extract on cartilage degradation in experimental osteoarthritis. Asian J Phytomed Clin Res 2015(3): 50-4. 